\title{
THE WULFF SHAPE AS THE ASYMPTOTIC LIMIT OF A GROWING CRYSTALLINE INTERFACE*
}

\author{
STANLEY OSHER ${ }^{\dagger}$ AND BARRY MERRIMAN ${ }^{\dagger}$
}

\begin{abstract}
We present a proof of a conjecture made in the field of crystal growth. Namely, for an initial state consisting of any number of growing crystals moving outwards with normal velocity given to be $\gamma(\vec{n})$, for $\vec{n}$ the unit outwards normal, then the asymptotic growth shape is a Wulff crystal, appropriately scaled in time. This shape minimizes the surface energy, which is the surface integral of $\gamma(\vec{n})$, for a given volume. The proof works in any number of dimensions. Additionally, we develop a new approach for obtaining the Wulff shape by minimizing the surface energy divided by the enclosed volume to the $\frac{1}{d}$ power in $R^{d}$. We show that if we evolve a convex surface (not enclosing a Wulff shape) under the motion described above, that the quantity to be minimized strictly decreases to its minimum as time increases. We have thus discovered a link between this surface evolution and this (generally nonconvex) energy minimization. A generalized Huyghen's principle is obtained. Finally, given the asymptotic shape we also obtain the associated (unique) convex $\gamma(\vec{n})$. The key technical tool is the level set method and the theory and characterization of viscosity solutions to Hamilton-Jacobi equations.
\end{abstract}

1. Introduction. The study of an anisotropic crystal growing in a melt gives rise to an equation relating the normal velocity of the motion to both the orientation of the crystal and to its curvature. In this paper we present a very simple and straightforward proof of a statement frequently made in the crystal literature - see e.g. Chernov [4], p. 215. Namely, in the case when the outwards normal velocity is equal to $\gamma(\vec{n})$, for $\gamma$ the surface tension and $\vec{n}$ the unit outwards normal, then the asymptotic growth shape is precisely the celebrated Wulff crystal, appropriately scaled in time. This shape minimizes the surface energy for a given volume.

The proof, which works in any number of space dimensions, is constructive, giving asymptotic in time estimates. Moreover, the initial state can consist of any number of growing crystals, some of which may even contain holes. As time increases the individuals will merge into a growing crystal whose asymptotic limit is a single Wulff shape.

The associated energy function need not be convex. Thus singularities in the shape, i.e. jumps in the normal direction, may develop not only in time but also in the asymptotic limit. Facets and other jumps in normal direction can be characterized precisely with the help of the theory of viscosity solutions $[5,6]$.

Numerical results using the localized level set method [21] and the high order essentially nonoscillatory approximations to Hamilton-Jacobi equations developed in $[14,15]$ validate our theoretical results. We shall discuss this in future work with $D$. Peng.

In a parallel work, Osher, Merriman, Zhao and Peng, [13], have developed a connection between the static shape of crystalline materials in the plane, and the propagation of shock waves. They show that there is a precise sense in which any two dimensional crystalline form can be described in terms of rarefaction waves and contact discontinuities.

\footnotetext{
*Received Oct 25, 1997; accepted for publication November 5, 1997. Research supported by DARPA/ONR N00014-92-J-1890, NSF DMS 94-04942 and a DARPA/NSF grant on Thin Films.

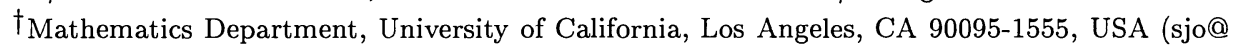
math.ucla.edu, barry@math.ucla.edu).
} 
We thus believe that there is an intimate and quantifiable link between the theory of crystalline shapes and the world of time dependent hyperbolic partial differential equations whose solutions develop jumps (or kinks) in finite time.

Additionally, in section III below, we develop a new approach to obtaining the Wulff shape by minimizing the surface energy divided by the enclosed volume to the $\frac{1}{d}$ power in $R^{d}$. We show that if we evolve a convex surface, (not enclosing a Wulff shape) under the motion described above, that the quantity to be minimized strictly decreases to its minimum as $t \rightarrow \infty$. Thus we have discovered a link between our HamiltonJacobi level set evolution with this (generally nonconvex) energy minimization.

Our first asymptotic result seems to have been first conjectured by Gross in 1918, [10]. Another important reference is Chernov [3]. There the author formally derives the partial differential equation for a closed curve in $R^{2}$ moving normal to itself with velocity $v(\theta), \theta$ is the angle of the normal. He parameterizes the curve as $\vec{R}(\theta, t)$ where $\vec{R}$ is the vector in $R^{2}$ connecting the origin to the point on the curve.

The differential equation for $\vec{R}$ is:

$$
\frac{\partial \vec{R}}{\partial t}=v(\theta) \vec{n}+v^{\prime}(\theta) \vec{n}^{\perp}
$$

where $\vec{n}$ is the outwards unit normal. He assumes steady state growth, $\vec{R}(\theta, t)=$ $\vec{R}_{0}(\theta) t$. Then (1.1) becomes:

$$
\vec{R}_{0}(\theta)=v(\theta) \vec{n}+v^{\prime}(\theta) \vec{n}^{\perp} .
$$

This is the formal Wulff construction when $v(\theta)=\gamma(\theta)$, the associated surface tension, see e.g. [11] and the original celebrated paper of Wulff [21].

Chernov's argument works only for a single curve and yields multivalued, swallow tailed type shapes if the associated energy is not convex. Moreover, he assume the existence of an asymptotic limit. The conjecture is valid in much more generality, with full rigor, as we shall show below.

An interesting related paper is by Soner [17]. There he uses the level set method of Osher and Sethian [14] as well as the theory of viscosity solutions, e.g. [5,6], both of which we also use, to analyze the large time asymptotics of the motion of a set. In Soner's work the normal velocity is a linear combination of the form

$$
v=\gamma(\vec{n})\left[a+b \partial_{E_{L}} \gamma(\vec{n})\right]
$$

Here $\partial_{E L} \gamma(\vec{n})$ is the Euler-Lagrange derivative of the surface tension function. The constant $b$ is positive and the associated level set based evolution equation is of the form (in $d$ dimensions)

$$
\frac{\partial \varphi}{\partial t}=|\nabla \varphi| \gamma\left(\frac{\nabla \varphi}{|\nabla \varphi|}\right)\left[a+b \sum_{i=1}^{d} \frac{\partial}{\partial x_{i}}\left[\gamma_{i}-\sum_{j=1}^{d} \frac{\gamma_{j} \varphi_{j} \varphi_{i}}{|\nabla \varphi|^{2}}+\gamma \frac{\varphi_{i}}{|\nabla \varphi|}\right]\right] .
$$

Here $\varphi$ is described below as a level set function associated with the interface, and $\gamma_{i}(\vec{n})=\frac{\partial}{\partial n_{i}} \gamma\left(n_{1}, \ldots, n_{d}\right)$. Also we define $|u|=\left(\sum_{i=1}^{d} u_{i}^{2}\right)^{\frac{1}{2}}$ for $u=\left(u_{1}, \ldots, u_{d}\right)$. Convexity of $\gamma$ is equivalent to the statement that the term multiplying $b$ is a nondecreasing function of the matrix $\left\{\frac{\partial^{2} \varphi}{\partial x_{i} \partial x_{j}}\right\}$. This means that the viscosity solution 
criterion is satisfied and this leads to well-posed initial value problems. Convexity is thus essential in Soner's work. In this context, convexity means that the function $|u| \gamma\left(\frac{u}{|u|}\right)$ is convex. Soner shows that if $a \leq 0$, any bounded curve has finite extinction time, and that if $a>0$ and the initial curve is large enough, it asymptotes to the Wulff shape.

Our problem will involve considering (1.4) for general nonconvex $\gamma$, with $b=0$. Nonconvexity means that discontinuities in the normal direction will develop.

In the next section we state and prove our main theorem. We do this by deriving an exact formula for the crystalline motion of a Wulff shape. We prove that such a shape moves in a self similar fashion. Then, for any initial shape bounding a region $\Omega$, we place a Wulff shape inside $\Omega$ and another, concentric, Wulff shape outside $\bar{\Omega}$. As $t$ increases, the two evolving self similar Wulff shapes trap the boundary of $\Omega$ between them. The desired result follows easily. We also provide extinction time estimates for inwards motion.

In section III we develop a new approach for obtaining the minimizing Wulff shape. Our procedure consists of starting with an arbitrary convex shape (in $R^{d}$ ) and evolving its boundary as above. We prove rigorously that the quantity to be minimized in the Wulff problem decreases as time increases to its minimum, achieved as $t \rightarrow \infty$, which occurs at the Wulff shape, and the quantity strictly decreases to its minimum in the event that the initial shape is not the minimizer. Thus we have discovered a new approach linking Hamilton-Jacobi level set evolution with (generally nonconvex) energy minimization. This technique appears to be applicable elsewhere.

A key step in the proof is the result that the location of the boundary of a set moving by normal velocity equal to a convex (in the sense of energy) $\gamma(\vec{n})$, at a later time $t$, is obtained through a generalized Huyghen's principle with the Wulff minimizing shape generalizing the usual spherical shape.

Moreover, we show that for a given asymptotic shape as $t \rightarrow \infty$, there is a unique convex (in the sense of energy) $\gamma$ such that this shape is the minimizer of the Wulff variational problem, and we give a formula involving a Legendre transform to obtain this $\gamma$.

2. Main results. We are considering the evolution of the boundaries of a family of hypersurfaces in $R^{n}$. Our initial region $\Omega \subset R^{n}$ is is the disjoint union of $\Omega_{i}$, compact sets with piecewise smooth boundary $\Gamma_{i}$. Each $\Omega_{i}$ is the closure of a nontrivial open set.. At each point at which the boundary is smooth, we move the hypersurface normal to itself with positive velocity $\gamma(\vec{n})$, where $\vec{n}$ is the unit outwards normal and $\gamma$ is uniformly continuous on the unit sphere.

Our procedure for analyzing this motion is based on the level set approach of Osher and Sethian [14], used also to analyze geometric motion elsewhere, e.g. [2,7,17]. We consider a Lipschitz continuous function $\varphi_{0}(x), \varphi_{0}: R^{n} \rightarrow R$ chosen so that

$$
\begin{array}{ll}
\varphi_{0}(x)<0 & x \varepsilon \Omega-\cup \Gamma_{i} \\
\varphi_{0}(x)=0 & x \varepsilon \cup \Gamma_{i}=\Gamma . \\
\varphi_{0}(x)>0 & x \notin \bar{\Omega}
\end{array}
$$

An example is $\varphi_{0}(x)=d(x, \Gamma)$, where $d$ is signed distance to the boundary, negative in the interior of the region and positive outside its closure.

The level set approach [14] applied here is simply to identify the evolution of the 
boundary at later times through solving the Hamilton-Jacobi equation

$$
\begin{aligned}
& \varphi_{t}=-\gamma\left(\frac{\nabla \varphi}{|\nabla \varphi|}\right)|\nabla \varphi|, \quad x \varepsilon R^{d}, t>0 \\
& \varphi(x, 0)=\varphi_{0}(x)
\end{aligned}
$$

where $H(\nabla \varphi)=|\nabla \varphi| \gamma\left(\frac{\nabla \varphi}{|\nabla \varphi|}\right)$ is the Hamiltonian, and identifying the zero level set of $\varphi(x, t)$ as $\Gamma_{t}$, the image of the surface under this motion. This works formally because the set of points $x(t)$ satisfying $\varphi(x, t) \equiv 0$ also satisfy

$$
\varphi_{t}+\dot{x} \cdot \nabla \varphi=0
$$

The normal component of velocity pointing in the outwards direction is

$$
\dot{x} \cdot \frac{\nabla \varphi}{|\nabla \varphi|}=\gamma(\vec{n})=\gamma\left(\frac{\nabla \varphi}{|\nabla \varphi|}\right)
$$

and thus 2.3) indicates that the zero level set (in fact all level sets) of $\varphi$ move according to $(2.2 \mathrm{a})$.

The theory of such Hamilton-Jacobi equations is included in the theory of viscosity solutions - see $[6,7]$. The solution generally develops kinks (jumps in the gradient) in finite time, even for smooth initial data. The viscosity solution criterion tells one (among other things) what these admissible jumps are - see e.g. [14]. A simple example occurs when $\gamma \equiv 1$ in (2.4). If $\varphi$ is initially signed distance to $\Gamma$, then the zero level set of the viscosity solution at time $t>0$ is just the $t$ level set of signed distance, e.g. [14]. It is not too hard to see that this generally develops kinks, e.g. if $\Gamma$ is a paraboloid. The entropy condition for this simple case [16] is just that we pick out the distance function, kinks and all. This was rigorized in [14], appealing to the analogous theory of shocks and the entropy condition occurring in conservation laws. There it was also shown that the motion is the limit obtained by adding a small multiple of the curvature of level set of $\varphi$ to (2.4) and letting this perturbation with this tangential dissipation go to zero. This is the type of motion we are generating. It agrees with classical motion whenever the hypersurface is smooth.

We now obtain our key result concerning the motion of a Wulff crystal. Let $r=\sqrt{x_{1}^{2}+x_{2}^{2}+\cdots+x_{d}^{2}}=|x|$ and $\nu=\frac{x}{r}$ be the radial and angle variables. The minimizing Wulff shape can be written (up to dilation) as

$$
r=W(\nu)
$$

where

$$
W(\nu)=\inf _{\substack{\nu \cdot \theta>0 \\|\theta|=1}} \frac{\gamma(\theta)}{(\nu \cdot \theta)}=\gamma^{*}(\nu)
$$

where $\nu \cdot \theta=\sum_{i=1}^{d} \nu_{i} \theta_{i}$, and $\gamma^{*}$ is the Legendre transform of $\gamma[8,18,19,20]$. Thus, the convexification of $\gamma$ can be obtained through

$$
\gamma^{* *}(\nu)=\max _{\substack{\nu \cdot \theta \geq 0 \\|\theta|=1}}(\nu \cdot \theta) \gamma^{*}(\theta)
$$


Frank's construction [9] associated with this is as follows: One considers the polar region

$$
r \leq R(\nu)=\frac{1}{\gamma(\nu)}
$$

and takes the outer convex envelope. This is the region

$$
r \leq \frac{1}{\gamma^{* *}(\nu)}
$$
for $\gamma$.

It is clear from (2.5) that the Wulff shape associated with $\gamma^{* *}$ is the same as that

We now present the main theorem from which our asymptotic results follow.

THEOREM 1. Consider (2.2) with initial data

$$
\varphi_{0}(x)=\frac{r}{W(\nu)} .
$$

The unique viscosity solution is

$$
\varphi(x, t)=\left[\frac{r}{W(\nu)}-t\right]^{+}
$$

where $x^{+}=\max (x, 0)$.

Proof. We use the second Hopf formula $[1,12]$ which gives the viscosity solution to $(2.2)$ for convex $\varphi_{0}(x)$.

The formula is:

$$
\varphi(x, t)=\max _{y \varepsilon R^{n}}\left(x \cdot y-\varphi_{0}^{*}(y)-t|y| \gamma\left(\frac{y}{|y|}\right)\right)
$$

where

$$
\varphi_{0}^{*}(y)=\sup _{y \varepsilon R^{n}}\left\{x \cdot y-\varphi_{0}(y)\right\}
$$

In polar coordinates, for our $\varphi_{0}$, we have

$$
\begin{aligned}
\varphi_{0}^{*}(r, \nu) & =\sup _{\substack{R \geq 0 \\
|\theta|=1}} R\left(r(\nu \cdot \theta)-\frac{1}{W(\theta)}\right) \\
& =\infty \text { if } r>\min _{\nu \cdot \theta>0}\left(\frac{1}{W(\theta) \nu \cdot \theta}\right)=\frac{1}{\gamma^{* *}(\nu)} \\
& =0 \text { if } r \leq \frac{1}{\gamma^{* *}(\nu)} .
\end{aligned}
$$

Thus

$$
\varphi(r, \nu, t)=\max _{\substack{R \gamma^{* *}(\theta) \leq 1 \\|\theta|=1}} R[r(\nu \cdot \theta)-t \gamma(\theta)]
$$

We have, since $\frac{1}{\gamma(\theta)} \leq \frac{1}{\gamma^{* *}(\theta)}$

$$
\max _{\substack{R \gamma(\theta) \leq 1 \\|\theta|=1}} R[r(\nu \cdot \theta)-t \gamma(\theta)] \leq \varphi(r, \nu, t) .
$$


The quantity on the left is maximized for each $\varphi$, either at $R=0$ or $R=\frac{1}{\gamma(\theta)}$. Thus

$$
\max _{\substack{|\theta|=1 \\ \nu \cdot \theta>0}}\left[r \frac{\nu \cdot \theta}{\gamma(\theta)}-t\right]^{+} \leq \varphi(r, \nu, t)
$$

or, from (2.5):

$$
\left[\frac{r}{W(\nu)}-t\right]^{+} \leq \varphi(r, \nu, t) .
$$

We also have, since $-t \gamma(\theta) \leq-t \gamma^{* *}(\theta)$

$$
\varphi(r, \nu, t) \leq \max _{\substack{R \gamma^{* *}(\theta) \leq 1 \\|\theta|=1}} R\left[r(\nu \cdot \theta)-t \gamma^{* *}(\theta)\right]
$$

Again, the maximum on the right, for each $\theta$, occurs either at $R=0$, or $R=\frac{1}{\gamma^{* *}(\theta)}$. Thus

$$
\varphi(r, \nu, t) \leq \max _{\substack{|\theta|=1 \\ \nu \cdot \theta>0}}\left[r \frac{(\nu \cdot \theta)}{\gamma^{* *}(\theta)}-t\right]^{+}
$$

and, from the fact that the Wulff shape associated with $\gamma * *(\theta)$ is the same as that for $\gamma(\theta)$, it follows that

$$
\varphi(r, \nu, t) \leq\left[\frac{r}{W(\nu)}-t\right]^{+} .
$$

This completes the proof.

We note that the viscosity solution to $(2.2)$ with $\varphi_{0}(x)=\frac{r}{W(\nu)}+c$, for any constant $c$ is just

$$
\varphi(r, \nu, t)=\left[\frac{r}{W(\nu)}-t\right]^{+}+c
$$

Thus we proceed as follows. Place a Wulff shape completely within the interior of $\Omega$. We may shift the origin so that there is a constant $a>0$ so that the zero level curve of

$$
\varphi_{i}(r, \nu, 0)=\frac{r}{W(\nu)}-a
$$

bounds a region which lies completely within $\Omega$. Next, we choose $b>0$ so that the zero level curve of

$$
\varphi_{0}(r, \nu, 0)=\frac{r}{W(\nu)}-b
$$

lies outside $\Omega$. Finally we construct a Lipschitz continuous function $\varphi_{\Omega}(r, \nu, 0)$ which is negative in the interior of $\Omega$, positive in the exterior of $\Omega$, vanishing on the boundary.

$$
\frac{r}{W(\nu)}-b \leq \varphi_{\Omega}(r, \nu, 0) \leq \frac{r}{W(\nu)}-a .
$$


Viscosity solutions to (2.2a) satisfy an ordering principle, so

$$
\left[\frac{r}{W(\nu)}-t\right]^{+}-b \leq \varphi_{\Omega}(r, \nu, t) \leq\left[\frac{r}{W(\nu)}-t\right]^{+}-a .
$$

This means that $\Gamma_{t}$, the zero level set of $\varphi_{\Omega}(\Omega, \nu, t)$, satisfies the following:

THEOREM 2. $\Gamma_{t}$ lies in the region

$$
W(\nu)\left(1+\frac{a}{t}\right) \leq \frac{r}{t} \leq W(\nu)\left(1+\frac{b}{t}\right) .
$$

This is our first main result.

Now we take the same family of hypersurfaces and move their boundaries inwards with normal velocity $\gamma(\vec{n})$. This motion is not generally reversible, even for Wulff shapes. However, it is for a Wulff shape corresponding to a convex $\gamma$.

This time we solve the Hamilton-Jacobi equation

$$
\varphi_{t}=\gamma\left(\frac{\nabla \varphi}{|\nabla \varphi|}\right)|\nabla \varphi|
$$

with initial data (2.9) as before. We now have, following the previous argument:

$$
\varphi(r, \nu, t)=\max _{\substack{R \gamma^{* * *}(\theta) \leq 1 \\|\theta|=1}} R[r(\nu \cdot \theta)+t \gamma(\theta)] .
$$

Following the argument used in (2.15), (2.16), we have

$$
\frac{r}{W(\nu)}+t \leq \varphi(r, \nu, t)
$$

Our upper bound for $\varphi$ follows from

$$
\begin{aligned}
\varphi(r, \nu, t) & =\max _{|\theta|=1}\left[\frac{r(\nu \cdot \theta)}{\gamma^{* *}(\varphi)}+t \frac{\gamma(\theta)}{\gamma^{* *}(\theta)}\right] \\
& \leq \frac{r}{W(\nu)}+t \kappa
\end{aligned}
$$

where

$$
1 \leq \kappa=\max _{|\theta|=1} \frac{\gamma(\theta)}{\gamma^{* *}(\theta)}<\infty
$$

Note $\kappa=1$ iff $\gamma=\gamma^{* *}$.

Thus, to estimate extinction time, we choose $\varphi_{i}(r, \nu, 0), \varphi_{0}(r, \nu, 0)$ as above and find

$$
\frac{r}{W(\nu)}+t-b \leq \varphi_{\Omega}(r, \nu, t) \leq \frac{r}{W(\nu)}+t \kappa-a
$$

This means that $\Gamma_{t}$ lies in the region

$$
(b-t)^{+} \geq \frac{r}{W(\nu)} \geq(a-t \kappa)^{+},
$$


which means that the extinction time, $t_{e}$, satisfies

$$
b \geq t_{e} \geq \frac{a}{\kappa}
$$

REMARK 2.1. Our results, Theorem 2 and (2.28) are valid for arbitrary initial regions having finite perimeter and nonempty interior, from the approximation results in [8] and the fact that the theory of viscosity solutions for Hamilton-Jacobi equations requires only bounded, uniformly continuous initial data.

Note added in proof. We have recently become aware of [22] by P. Soravia. In section 3 of that paper the author gives a different proof of our Theorem 2. Additionally, we recently noticed that the work of J. Gravner and D. Griffeath, [23], concerning cellular automata contains a result which is related to our Theorem 2, although the authors did not explicitly draw this conclusion.

3. A new approach to obtaining the minimizing Wulff shape. In this section we discuss the link between the crystalline motion described above and a new approach to minimizing the (generally nonconvex) energy associated with the Wulff shape.

In $R^{d}$ we obtain the Wulff shape, up to scaling, by minimizing the quantity:

$$
E(\Gamma)=\frac{A}{V^{1-1 / d}}
$$

where $\Gamma$ is the boundary of a convex set $\Omega, V$ is its volume and $A$ is the surface integral of $\gamma(\vec{n})$, i.e. the energy.

We shall show that moving $\Gamma$ normal to itself with velocity $\gamma(\vec{n})$, as described in section II, strictly diminishes $E(\Gamma(t))$ unless $\Gamma(t)$ is a Wulff shape, in which case $E(\Gamma(t))$ is constant in time. We obtain the proof below. Since this is true, then our main result, Theorem 2 , implies that the Wulff shape is the unique minimizer since $E(\Gamma(t)) \rightarrow E\left(\Gamma_{W}\right)$ as $t \rightarrow \infty$. (It is well known that we need only consider convex $\Gamma$ in our minimization procedure).

We compute, for such a motion, $\frac{\partial}{\partial t} E(\Gamma(t))$. It is a simple observation that $\frac{\partial V}{\partial t}=$ $A$, so we have

$$
\frac{\partial}{\partial t} E(\Gamma(t))=d \frac{\partial^{2}}{\partial t^{2}}\left(V(\Gamma(t))^{\frac{1}{d}}\right.
$$

Thus we are trying to prove that, under this motion, $(V(\Gamma(t)))^{\frac{1}{d}}$ is a strictly concave function of $t$, unless $\Gamma(t)$ bounds a Wulff shape, in which case $V(\Gamma(t))^{\frac{1}{d}}$ is affine in time.

The second statement follows simply from Theorem 1 . If the initial shape $\Gamma(0)$ is determined by:

$$
r=W(\nu)
$$

then $V(t)$ is:

$$
r=W(\nu)(1+t)
$$

so

$$
V(\Gamma(t))=\frac{1}{d}(1+t)^{d} \int_{\Gamma(0)} W(\nu) d \nu
$$


Thus $(V(\Gamma(t)))^{\frac{1}{d}}=(1+t) c$, for $c$ constant.

We wish to show

$$
V \frac{\partial^{2} V}{\partial t^{2}} \leq\left(1-\frac{1}{d}\right)\left(\frac{\partial V}{\partial t}\right)^{2}=\left(1-\frac{1}{d}\right) A^{2}
$$

with strict inequality holding unless $\Gamma(t)$ is a Wulff shape.

We note that in the special case $\gamma \equiv 1$, when the Wulff shape is a sphere, (3.4) reduces to showing

$$
\begin{aligned}
& \text { (Volume enclosed in } \Gamma(t)) \text { (Surface integral of mean curvature) } \\
& \leq\left(1-\frac{1}{d}\right)(\text { surface area } \Gamma(t))^{2}
\end{aligned}
$$

with equality holding iff $\Gamma(t)$ is a sphere. This is the well known isoperimetric inequality.

More generally we note that $\frac{\partial^{2} V}{\partial t^{2}}$ is the surface integral of $\gamma(\vec{n})$ times the divergence of $[\gamma(\vec{n}) \vec{n}+$ tangential gradient of $(\gamma(\vec{n}))]$.

Following the notation of (1.4), with $\delta$ the Dirac delta function, $\vec{n}=\frac{\nabla \varphi}{|\nabla \varphi|}$, and $H$ the Heaviside function, we must show the following inequality involving volume integrals:

$$
\begin{aligned}
\int(1-H(\varphi)) & \int \delta(\varphi)|\nabla \varphi| \gamma(\vec{n})\left[\nabla \cdot\left[D_{\vec{n}} \gamma(\vec{n})-\left(\vec{n} \cdot D_{\vec{n}} \gamma\right) \vec{n}+\gamma \vec{n}\right]\right] \\
\leq & \left(1-\frac{1}{d}\right)\left(\int \gamma(\varphi)|\nabla \varphi| \gamma(\vec{n})\right)^{2}
\end{aligned}
$$

In words, we must show:

(Volume enclosed by $\Gamma(t)$ ) (Surface integral

$$
\begin{aligned}
& \text { over } \left.\Gamma(t) \text { of } \gamma(\vec{n}) \text { times } \partial_{E L} \gamma(\vec{n})\right) \\
& \quad \leq\left(1-\frac{1}{d}\right)(\text { Energy of } \Gamma(t))^{2}
\end{aligned}
$$

We know that the convexification of $\gamma$ satisfies $\gamma^{* *}(\vec{n}) \leq \gamma(\vec{n})$. Moreover, for a fixed convex $\Gamma(t)$

$$
\gamma^{* *}(\vec{n})<\gamma(\vec{n})
$$

means that $\partial_{E_{L}} \gamma(\vec{n})<0$.

Thus

$$
\gamma(\vec{n}) \partial_{E_{L}} \gamma(\vec{n}) \leq \gamma^{* *} \partial_{E_{L}} \gamma^{* *}(\vec{n})
$$

So we have the following interesting result:

LEMMA 3.1. Inequality (3.6) is valid if it is valid for $\gamma$ replaced by $\gamma^{* *}$, the convexified surface tension.

Thus we need only show that $\left(V(\Gamma(t))^{\frac{1}{d}}\right.$ is a strictly concave function of $t$ for convex non Wulff shapes evolving under a convex velocity $\gamma(\vec{n})$.

For the evolving shape we solve (2.2) with initial data

$$
\varphi_{0}(x)=\varphi_{0}(r \nu)=\frac{r}{\psi(\nu)} .
$$

The boundary, $\Gamma(0)$, is the 1 level set of $\varphi_{0}(x)$

$$
r=\psi(\nu) \text {. }
$$


According to the first Hopf formula for convex Hamiltonians [1,12], the solution is

$$
\varphi(x, t)=\min _{w \varepsilon R^{d}}\left\{\varphi_{0}(x-t w)+t H^{*}(w)\right\}
$$

where $H^{*}(w)$ is the Legendre transform of our Hamiltonian $H(w)=w \gamma\left(\frac{w}{|w|}\right)$

$$
\begin{aligned}
H^{*}(w) & =\sup _{w \varepsilon R^{d}}\left\{y \cdot w-t w \gamma\left(\frac{w}{|w|}\right)\right\} \\
& =0 \text { if } r \leq W(\nu) \\
& =\infty \text { if } r>W(\nu)
\end{aligned}
$$

Thus

$$
\varphi(x, t)=\min _{\substack{w=R \theta \\ \text { with } R \leq W(\theta)}} \varphi_{0}(x-t w)
$$

This means that $\Gamma(t)$ bounds the convex region $\Omega(t)$ defined by

$$
\Omega(t)=\Omega(0) \oplus t W
$$

where $t W$ is the Wulff shape $r \leq t W(\nu)$.

The sum $\oplus$ in (3.12) means that

$$
z \varepsilon \Omega(t) \Leftrightarrow z=x+y, x \varepsilon \Omega, y \varepsilon t W .
$$

We wish to show that the quantity $(V(\Omega(t)))^{\frac{1}{d}}$ is concave in $t$, i.e.

$$
\left(V\left(\Omega\left(\theta t_{1}+t(1-\theta) t_{2}\right)\right)^{\frac{1}{d}} \geq \theta\left(V\left(\Omega\left(t_{1}\right)\right)\right)^{\frac{1}{d}}+(1-\theta)\left(V\left(\Omega\left(t_{2}\right)\right)\right)^{\frac{1}{d}}\right.
$$

for $0 \leq \theta \leq 1, \quad t_{1}, t_{2} \geq 0$.

Now using (3.12), we claim

$$
\begin{aligned}
\Omega\left(\theta t_{1}+(1-\theta) t_{2}\right) & =\Omega(0) \oplus\left(\theta t_{1}+(1-\theta) t_{2}\right) W \\
& =\theta\left(\Omega(0) \oplus t_{1} W\right) \oplus(1-\theta)\left(\Omega(0) \oplus t_{2} W\right) .
\end{aligned}
$$

We need only show that points of the form $z+\theta t_{1} \bar{x}+(1-\theta) t_{2} \bar{y}$ for $\bar{x}, \bar{y} \varepsilon W(\nu)$ and $z \varepsilon \Omega(0)$, are exactly of the form:

$$
\theta\left(x+t_{1} \bar{x}\right)+(1-\theta)\left(y+t_{2} \bar{y}\right)
$$

for $x, y$ in $\Omega(0)$. This follows precisely because $\Omega(0)$ is convex.

Thus, we have shown:

$$
\Omega\left(\theta t_{1}+(1-\theta) t_{2}\right)=\theta \Omega\left(t_{1}\right) \ominus(1-\theta) \Omega\left(t_{2}\right)
$$

and (3.13) follows from the Brunn-Minkowski inequality.

Thus, we have our second main result: 
THEOREM 3.1. Given an arbitrary initial convex shape $\Omega(0)$ bounded by $\Gamma(0)$ which is piecewise smooth. Let the boundary move outward normal to itself with velocity $\gamma(n)$ in the Hamilton-Jacobi level set sense of section 2. Then

$$
E(\Gamma(t))=\frac{A(\Gamma(t))}{(V(\Gamma(t)))^{1-\frac{1}{d}}}
$$

decreases to that of the minimizing Wulff shape and is strictly decreasing unless the initial shape is a Wulff shape.

COROLlary 3.1. The Wulff shape is the minimizer of $E(\Gamma)$ for any continuous $\gamma(\vec{n})$ and the minimum can be taken over bounded measurable sets with finite perimeter.

The proof of this involves realizing that (1) we need only consider convex sets in our minimization procedure and (2) the assumption of piecewise smooth boundary, which was needed only in the step where we replace $\gamma$ by its convexification, is adequate, by the approximation results for $B V$ characteristic functions mentioned in [8].

COROLlary 3.2. The motion of the boundary of a general set with a finite perimeter and nonempty interior for convex $\gamma(\vec{n})$ is given by (3.12).

Thus the Wulff shape acts as an elemental basis for moving the boundary of a set, and conversely given a convex shape

$$
r \leq W(\nu)
$$

we may find a convex $\gamma$

$$
\gamma(\nu)=\max _{\substack{\nu \cdot \theta>0 \\|\theta|=1}}(\nu \cdot \theta) W(\theta)
$$

which is the normal velocity corresponding to the motion generated by (3.12).

COROLlary 3.3. Given that the asymptotic motion of an arbitrary family of hypersurfaces in the sense of Corollary 3.2, satisfies Theorem 2, then the resulting shape $r=W(\nu)$ is the minimizer of $E(\Gamma)$ in (3.1), where the unique convex surface tension function $\gamma(\vec{n})$ is defined by:

$$
\gamma(\nu)=\max _{\substack{\nu \cdot \theta \geq 0 \\|\theta|=1}} W(\theta)
$$

\section{REFERENCES}

[1] M. Bardi And L.C. Evans, On Hopf's Formulas for Solutions of Hamilton-Jacobi Equations, Nonlinear Anal. TMA, v. 8, (1984), pp. 1373-1381.

[2] Y.G. Chen, Y. Giga, And S. Goto, Uniqueness and Existence of Viscosity Solutions of Generalized Mean Curvature Flow Equations, J. Diff'l. Geom., v. 33, (1991), pp. 749786.

[3] A.A. Chernov, The Kinetics of the Growth Form of Crystals, Soviet Physics Crystallography, v. 7, (1963), pp. 728-730, translated from Krystallografiya, v. 7 (1962), pp. 895-898.

[4] A.A. Chernov, Modern Crystallography III, Crystal Growth, Springer-Verlag, Berlin, (1984).

[5] M.G. Crandall, H. Ishil and P.-L. Lions, User's Guide to Viscosity Solutions of Second Order Partial Differential Equations, Amer. Math. Soc. Bull., v. 27, (1992), pp. 1-67.

[6] M.G. Crandall and P.-L. Lions, Viscosity Solutions of Hamilton-Jacobi Equations, Trans. Am. Math. Soc., v. 277, pp.1-42.

[7] L.C. Evans And J. Spruck, Motion of Sets by Mean Curvature, I, J. Diffl. Geom., v. 33, (1991), pp. 635-681. 
[8] I. FonsecA, The Wulff's Theorem Revisited, Proc. Royal Soc. London A, v. 432 (1991), pp. $125-124$.

[9] F.C. Frank, On the Kinematic Theory of Crystal Growth and Dissolution Processes. Growth and Perfection of Crystals, in Growth and Perfection of Crystals, ed. R.H. Doremus, B.W. Roberts, D. Turnbull, John Wiley, New York (1958).

[10] R. Gross, Zur Theorie des Washstrums und Lösungsforganges Kristalliner Materie, Abhandl. Math.-Phys. Klasse Köngl. Sächs, Wiss, v. 35, (1918), pp. 137-202.

[11] M.E. Gurtin, Thermomechanics of Evolving Phase Boundaries in the Plane, Oxford Mathematical Monographs, Clarendon Press, Oxford (1993).

[12] E. Hopf, Generalized Solutions of Nonlinear Equations of First Order, J. Math. Mech., v. 14, (1965), pp. 951-973.

[13] S. Osher, B. Merriman, H.-K. Zhao and D. Peng, The Wulff Crystal Shape as the Solution to Riemann Problems for Scalar Conservation Laws, (1997) preprint.

[14] S. Osher And J.A. Sethian, Fronts Propagating with Curvature Dependent Speed: Algorithms Based on Hamilton-Jacobi Formulations, J. Comput. Phys., v. 79, (1988) pp. 12-49.

[15] S. Osher AND C.-W. SHU, High-Order Essentially Nonoscillatory Schemes for HamiltonJacobi Equations, SIAM J. Numer. Anal., v. 28, (1991), pp. 907-922.

[16] J.A. Sethian, Curvature and Evolution of Fronts, Comm. Math. Phys., v. 101, (1985), pp. 487-499.

[17] H.M. Soner, Motion of a Set by the Curvature of Its Boundary, J. Diff. Eqn., v. 101, (1993) pp. 313-372.

[18] J. TAYLOR, Existence and Structure of Solutions to a Class of Non Elliptic Variational Problems, Sympos. Math., v. 14, (1974), pp. 499-508.

[19] J. TAYLOR, Unique Structure of Solutions to a Class of Non Elliptic Variational Problems, Proc. Sympos. Pure Math., v. 27, (1975), pp. 419-427.

[20] G. WulfF, Frage der Geschwindigkeit des Wachstums und der Anflosung der Krystallflachen, Z. Krystall. Min, v. 34, (1901), pp. 449-530.

[21] H.-K. Zhao, M. Kang, B. Merriman, D. Peng and S. Osher, A PDE Based Reinitialization for the Fast, Localized Level Set Method, (1997), preprint.

[22] P. Soravia, Generalized Motion of a Front Propagating Along Its Normal Direction: a Differential Games Approach, Nonlinear Analysis, TMA, v. 22, (1994), pp. 1247-1262.

[23] J. Gravner and D. Griffeath, Threshold Growth Dynamics, AMS Transactions, v. 340, (1993), pp. 837-870. 